\title{
Advanced Oceanic Power Harvesting Systems for Autonomous Undersea Sensors
}

\author{
John Hickin ${ }^{a}$, Elena Busuttil ${ }^{a}$ and Amir Anvar ${ }^{a}$ \\ School of Mechanical Engineering, \\ The University of Adelaide, \\ Adelaide, South Australia 5005 \\ AUSTRALIA \\ Emails: hickin88@gmail.com ; elena.busuttil@student.adelaide.edu.au; amir.anvar@adelaide.edu.au
}

\begin{abstract}
The technologies behind autonomous underwater sensory systems are continuously developing resulting in increasing capabilities and operating times. Power supply to these autonomous sensors is commonly facilitated via the use of primary alkaline cells, which causes limitations to operating periods. This study aimed to conduct a feasibility study into the application of small scale power generation to supplement power requirements for underwater autonomous sensory equipment.

An assessment of the availability and potential of various forms of energy which may be harvested within the oceanic environment resulted in wave energy being the most suitable form of harvestable energy. With waves as the chosen energy source, two wave energy harvesting devices, a surface buoy energy converter and a heaving point absorber were modelled and developed.
\end{abstract}

The surface buoy energy converter design aims to harvest energy from all six degrees of freedom induced from multidimensional dynamic conditions that exist on the surface of the ocean through the use of linear generators.

The heaving point absorber is a sub-surface operating device which incorporates a ball screw mechanism to translate vertical displacement of the waves to rotary motion for utilisation of a rotary generator.

Both devices require conversion of the generated power to a useable form for an underwater sensor. For each device an electrical system has been designed to ensure continuous power to the sensors. The status and performance of the devices can be monitored through the use of a data logger with remote communications.

Manufacture of prototype devices was undertaken to allow laboratory testing. The devices were tested for watertightness, proof of concept and power generation. These tests successfully proved both concepts and allowed for recommendations into further design improvements. The power production during the testing revealed modest power production of the surface buoy energy converter and a peak power production of approximately 25 Watts from the heaving point absorber.

Keywords: Energy, Converter, Wave Energy Harvesting Simulation, Ocean, Sensors, Automation 


\section{INTRODUCTION}

Interest into alternative methods of power generation through the use of power harvesting devices has resulted in the need for the determination of a suitable energy source within the operational environment. Determination of a suitable energy source within the environment will enable the generation of design constraints for prototype design.

\section{BACKGROUND}

The concept of harvesting energy from the ocean into a usable form has been cogitated for considerable time. Patents existed as early as 1799 in relation to the extraction of useful energy from waves (Clement et al. 2002). Since humans began to postulate using energy from the ocean as a potential energy source for power generation, many different harvesting designs have been patented.

In more recent times such as the peak oil crisis during 1973, a surge of research and development into the exploitation of wave energy in Europe (Clement et al. 2002) has begun. Since the initial developments, there has been a period of stagnation, with a revival due to the development of renewable energies to reduce carbon footprints (Behrens et al. 2011). Recent developments make the exploitation of wave and current energy closer than ever before, with a number of commercial plants in Australia, Europe, Israel and elsewhere, operational or within the construction phase (Clement et al. 2002). Applications of smaller scale power generation have seen progression and have been fuelled by the need for power supplementation of autonomous oceanic sensory devices. The first commercial production of electricity occurred in 2000 by the company Wavegen, with a generator output of 500kW (Aubrecht 2005).

\section{OCEAN ENERGY SOURCES}

Sources of oceanic energy come in an array of different forms and include tidal currents, marine currents, geothermal vents and waves, which are all at least partially concentrated forms of gravitational or solar energy (Vining 2005). Taking into account the operational environment of the intended sensor deployment area, from which an energy harvesting device must be capable of extracting a minimum power generation target of 20 Watts, waves were found to be the most viable energy form.

\subsection{Wave Energy}

Wave energy is generally a result of the transfer of energy from solar to wind and finally to wave energy. The conversion of solar energy to wind energy results in a concentration of time averaged power flow from 0.1$0.3 \mathrm{~kW} / \mathrm{m}^{2}$ from the horizontal surface to $0.5 \mathrm{~kW} / \mathrm{m}^{2}$ of hypothetical area normal to the wind direction (Falnes 2007). Falnes (2007) also notes that the concentration of wind energy to wave energy results in a typical average power flow intensity of $2-3 \mathrm{~kW} / \mathrm{m}^{2}$, perpendicular to the wave direction. This natural concentration of energy results in an enormous source of renewable energy with the benefit of having smaller stagnant periods of energy generation as compared with solar and wind (Falnes 2007). Although the energy within waves is quite substantial, there is a large variability in power levels. This level of variability in wave conditions is further amplified in coastal waters due to wave refraction, reflection, diffraction and bottom friction (Falnes 2007). The yearly average high wave power level of a particular offshore location can differ by a factor of two as compared with the yearly average low (Torsethaugen 1982).

A snapshot of an oceanic environment will reveal several different waves that propagate from different events, with different wavelengths and directions (Falnes 2007). For an energy harvesting device, it is important that the device is capable of converting energy from the prevalent moderate waves whilst withstanding the extreme stormy seas that contribute significantly to the average wave energy figures (Falnes 2007).

Waves within the Southern Australian Ocean are well recognised by their large size (Behrens et al. 2011). Figure 1 shows the comparatively large amount of wave energy that exists off the southern coastal regions of Australia. The wave power, per unit of wavefront of a region is given approximately by the following equation (Herbich 2000):

$$
P_{\text {wavefront }}=\frac{\rho_{\text {water }} g^{2}}{64 \pi} H_{s}^{2} T \approx 0.5 T H_{s}^{2}
$$


Where $\rho_{\text {water, }}$ is the sea water density, $g$, is the gravitational constant, $T$, the wave period and $H_{s}$, the significant wave height. Figure 1 highlights elevated levels of wave energy density off the south coast of Australia.

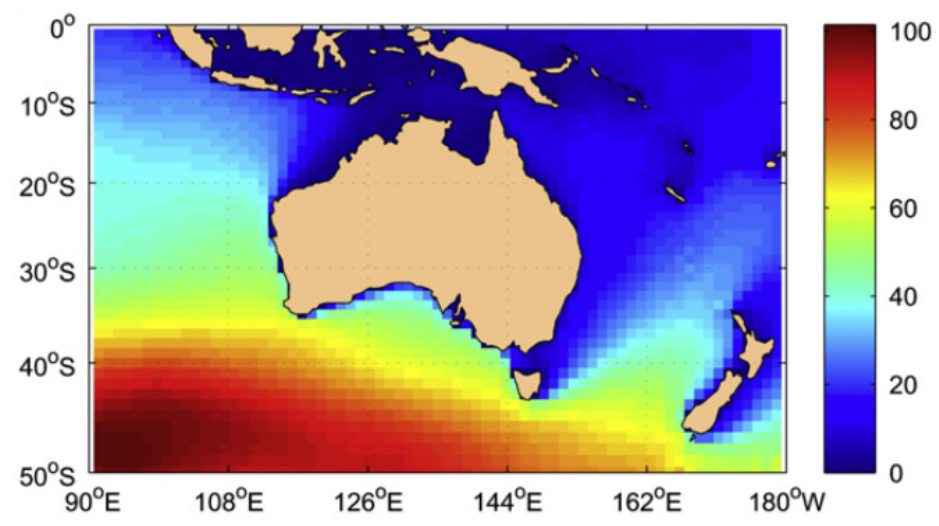

Figure 1. Wave energy Flux $P_{\text {wavefront }}(\mathrm{kW} / \mathrm{m})$ (Behrens et al. 2011 pp. 212)

The wave energy per unit area can be represented by the following equation (Falnes 2007)

$$
E_{\text {density }}=\frac{\rho_{\text {water }} g H_{s}^{2}}{16}=\rho_{\text {water }} g \int_{0}^{\infty} S(f) d f
$$

Where $\rho_{\text {water }}$ is the density of seawater, $g$ is the acceleration due to gravity, $H_{s}$ is the significant wave height and $S(f)$ is the wave spectrum, measured in $\mathrm{m}^{2} / \mathrm{Hz}$. The equation includes the energy associated with the kinetic energy and potential energy and assumes that surface tension effects are negligible.

A vertical plane with a unit width orthogonal to wave propagation has a wave energy flux that can be represented in terms of Equation 3 by the following relationship (Phillips 1977),

$$
P_{\text {wavefront }}=c_{g} E_{\text {density }}
$$

where $c_{g}$ is equal to the group velocity which represents the energy transport velocity.

The omni-directional (gross) wave energies of an environment decline with decreasing distance to shore (Folley and Whittaker 2009). Figure 2 shows the reduction average directionally-resolved (net) wave power for a sea state with an initial power level of $50 \mathrm{~kW} / \mathrm{m}$ and an energy period of 10

Wave energy below the surface of the water decays according to a factor of $e^{-2 \pi d / \lambda}$, where $\mathrm{d}$ and $\lambda$ represent the depth and wavelength respectively (Behrens et al. 2011). Energy harvesting devices designed to extract energy from the surface of the ocean are exposed to a hostile operating environment. The option of having a submerged energy harvesting device results in a reduced potential for damage to the equipment, due to the reduced energy within the devices operational environment.

\section{ENERGY HARVESTING DEVICE MODELLING}

Preliminary concept designs were developed with the aim of facilitating the supplementation of power to underwater sensor systems. Through the evaluation and modelling of the concept designs against a set of design criteria, two models with different mechanism operations were identified for further development; 
- Surface buoy energy converter: Surface operating device, incorporating a number of permanent magnet linear generators within a buoy. This orientation will harvest energy from all six degrees of freedom as experienced by the multidimensional dynamic conditions that exist on the surface of the ocean.

- Heaving point absorber: Subsurface operating device, incorporating a ball screw mechanism to translate the vertical displacement of the waves into the rotary motion necessary to power a rotary generator.

\subsection{Surface Buoy Energy Converter}

The surface buoy energy harvesting device utilises the multidimensional "choppy" conditions that commonly exist on the ocean surface.

The surface buoy, as shown in Figure 3, features an internal framework to seat four permanent magnet linear generators. Three of the generators are placed horizontally and one is situated vertically. Placement of the linear generators has been optimised to ensure exploitation of translational and pitching motions as a result of multidirectional ocean surface motions. The surface buoy features a submersed body which consists of a frame and container which allows for the housing of battery management and system diagnostics equipment. The device incorporates a non-concentric centre of gravity which allows the device to locate vertically whilst allowing suitable amounts of horizontal translation and pitching motions.

The surface buoy energy converter was manufactured almost entirely using a $3 \mathrm{D}$ printing process which enabled the production of a relatively complex internal structure that would otherwise be very expensive to produce, whilst still using a suitable and robust material.

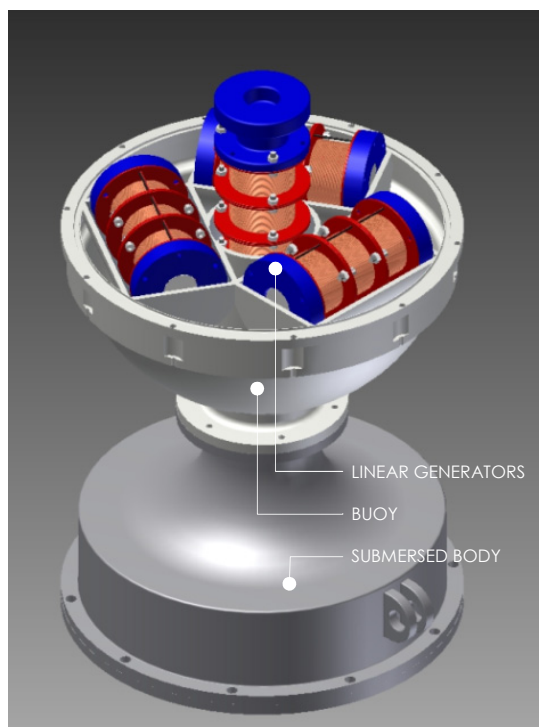

Figure 3. Surface buoy energy converter with top cover removed, revealing linear generator orientations

\subsection{Heaving Point Absorber}

The heaving point absorber design operates below the surface of the water, utilising the vertical translation due to swell within the ocean as seen in figure 4 . The device is optimised to function at about 10 metres below the surface of the ocean, where its survivability is increased due to reduced amounts of energy experienced within its operational environment. The hydrostatic pressure at this depth acting on the top of the shaft provides the force necessary to return the shaft during the downward heaving motion of a wave. A surface buoy provides the buoyancy force necessary to raise the shaft during the upward heaving motion. Mooring is used to vertically locate the device and maintain a constant depth below the surface of the ocean.

To translate the linear heaving motion to rotary motion for use by a rotary generator, the design utilises a high lead ball bearing nut and screw mechanism. The mechanism operates by restricting

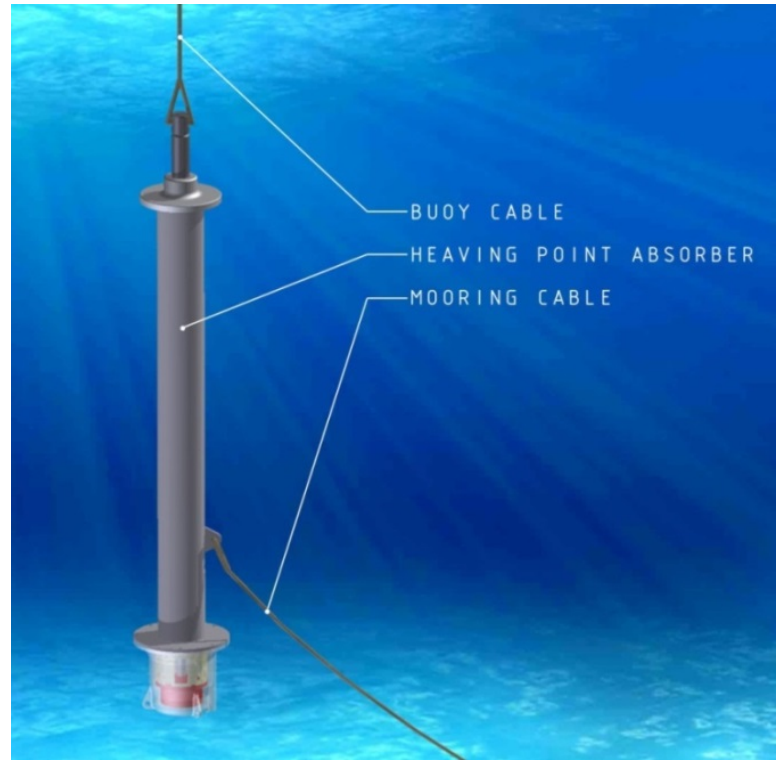

Figure 4. Heaving point absorber the motion of the nut to vertical translation which induces rotary motion of the screw. To ensure that the nut experiences only vertical translational motion, a linear rail guide was incorporated into the design as seen in Figure 5. The screw rotates in both directions depending on the direction of the vertical motion of the nut. As a result, a rotary generator with the capability to generate electricity in both rotational directions was salvaged from a commercially available wind turbine. Consideration of the pitch of the device was needed to ensure that the screw had the correct lead to match recommended operating speeds of the rotary generator. The pitch selection was optimised for waves between 1 and 2 metres using maritime wave data whilst a screw rotational speed of 600 revolutions per minute was assumed. The pitch of the screw was calculated to 
be between 31.6 to 42.4 millimetres which allowed the design of the device utilising an off the shelf high pitch ball bearing nut and screw.

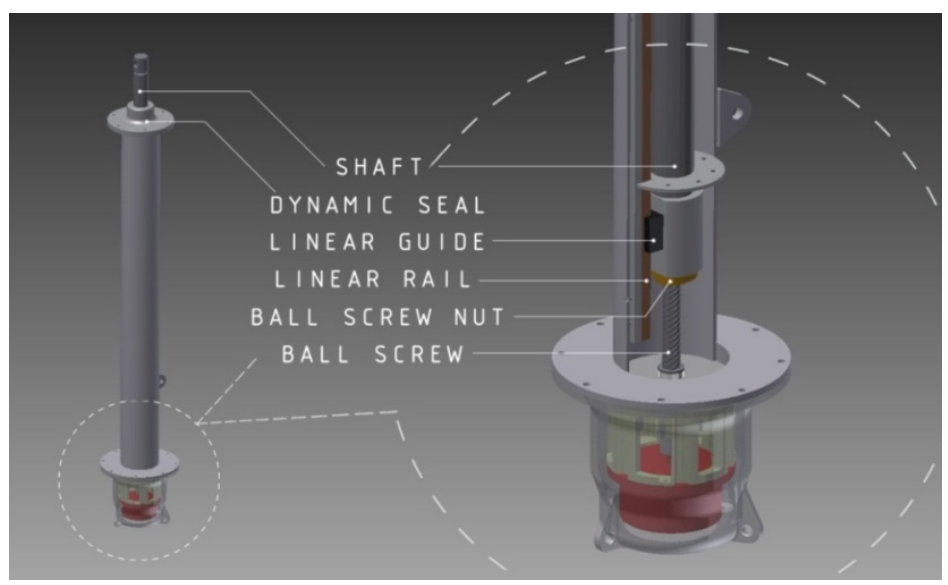

Figure 5. Heaving point absorber internal design

Major components of the device were designed and manufactured using commercially available aluminium tube and flat plate. A number of components were 3D printed to reducing manufacture time and cost.

\section{ELECTRICAL AND AUTOMATION SYSTEM MODEL}

An electrical and automation system was designed and manufactured for each of the energy harvesting device designs. These designs are tailored to each of the devices to optimise the power output, ensure continuous power to the sensor and the gather and transmit system diagnostics information.

Each electrical system incorporates a number of components allowing the devices to convert the mechanical energy experienced by the device into electrical energy to be used by the sensor. The current must first be rectified from an alternating to a direct current. Then through the use of a charge controller with dump resistors and a rechargeable battery system, consisting of 2 rechargeable batteries, ensures that there is continuous power to the sensor and diagnostic equipment. The Fuzzy Expert System (IF...THEN...) rules model was used for automatic switching of energy to control the rechargeable battery system. The following is an example of the rules of the system:

Rule 1; IF: $11 \mathrm{v}<$ Battery $1<12 \mathrm{v} \& 11 \mathrm{v}<$ Battery $2<12 \mathrm{v}$ THEN: [No Change].

Rule 2; IF: $10 \mathrm{v}<$ Battery $1<10.9 \mathrm{v} \& 11 \mathrm{v}<$ Battery $2<12 \mathrm{v}$ THEN: Slow-Switch [Battery 1 TO Battery 2].

Rule 3; IF: $0 \mathrm{v}<$ Battery $1<9.9 \mathrm{v} \& 11 \mathrm{v}<$ Battery $2<12 \mathrm{v}$ THEN: Fast-Switch [Battery 1 TO Battery 2 AND Charge Battery 1] and so on. This ensures that there is continuous power to the sensor.

The system also features a data logger to record the status and performance of the device. The data logger was initially used to gather testing results but was selected with the possibility of adding a remote communications to allow for to the minute status information for the device in operation.

\section{LABORATORY TESTING}

Power generation testing was conducted in a 6 metre water tank, with each device subjected to conditions that are expected within the intended operational areas.

\subsection{Power Generation: Surface Buoy Energy Converter}

Simulation of its operation in choppy oceanic conditions was conducted through manually inducing a rocking motion into the buoy. The first test was conducted using a weighted pipe attachment to simulate the attachment of the submerged body as seen in Figure 6.

For the second test the buoy, without the submerged body, used a flat plate cover over the bottom flange section of the surface buoy. By viewing the results from the data logger, it was clear that the device had a significantly greater power generation capability without the submerged body attached. The movement

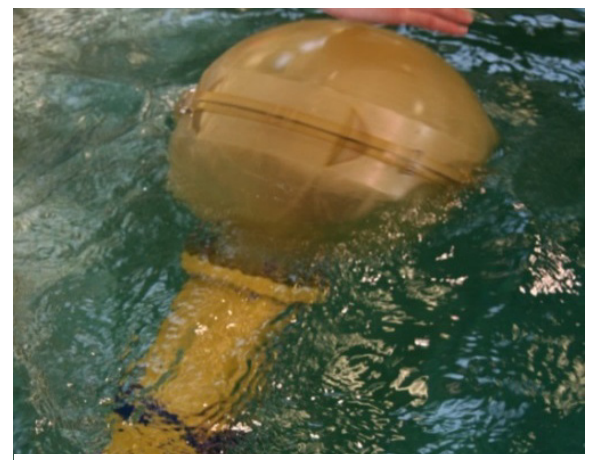

Figure 6. Surface buoy energy converter with submerged body attached 
of the device was observed to have much greater irregularity due to a more central centre of gravity location. This resulted in a greater amount of kinetic energy transfer between the surface buoy and the internal magnets in the linear generators.

Due to the low current and voltage figures obtained with inadequate resolution from the data logger, the actual power production of the device could not be accurately measured. However, it was discovered that the vertical linear generator produced the highest current, but in general the linear generators were the main contributors to the lower current figures and would need further design iterations if an increase in power production is required. This device may have application in small scale power production where the reduction of weight, size and ease of distribution of the device are the primary concern.

\subsection{Power Generation: Submerged Heaving Point Absorber}

Testing of the device involved submerging the device with a weight connected to the bottom of the device as seen in Figure 7. After the device was deemed watertight, testing was conducted to simulate a 4 second and a 2 second shaft extension time which equates to vertical velocities $0.5 \mathrm{~m} / \mathrm{s}$ and $0.25 \mathrm{~m} / \mathrm{s}$ respectively. The top of the shaft extended up from a 3.5 metre water depth with the retraction of the main shaft taking around 5 seconds. As the device is intended to operate at a water depth of around 10 metres, the hydrostatic pressure on top of the shaft a this depth would result in the reduction of the retraction time during the downward heaving portion of the wave.

The current production associated with an upward extension and retraction of the main shaft is shown in figure 8 . Extension time is 2 seconds initiating at 12:01:39 and finishing at 12:01:41 and the retraction time is 5 seconds initiating at 12:01:43 and finishing at 12:01:48. Figure 8 also reveals a higher current production during the extension of the main shaft compared with its retraction due to a higher upward velocity of the shaft.

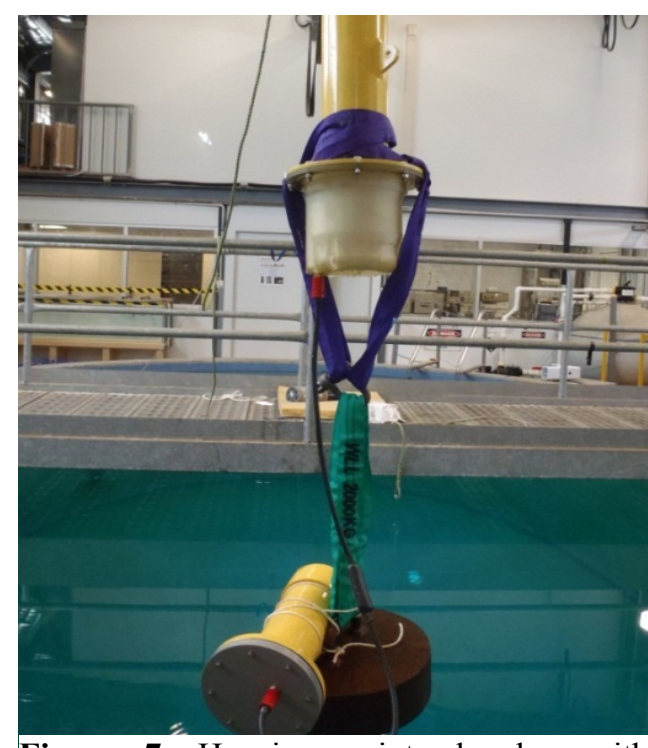

Figure 7. Heaving point absorber with weight attached and external electrical housing

Voltage and current measurements allowed the calculation of power generation. Instantaneous power generation values were observed to be as high as 25 Watts. Using this data a more realistic power generation value of 10 Watts was calculated by averaging the instantaneous power generation of one bi-directional stroke over the stroke time period, with the device submerged to its correct operating depth.

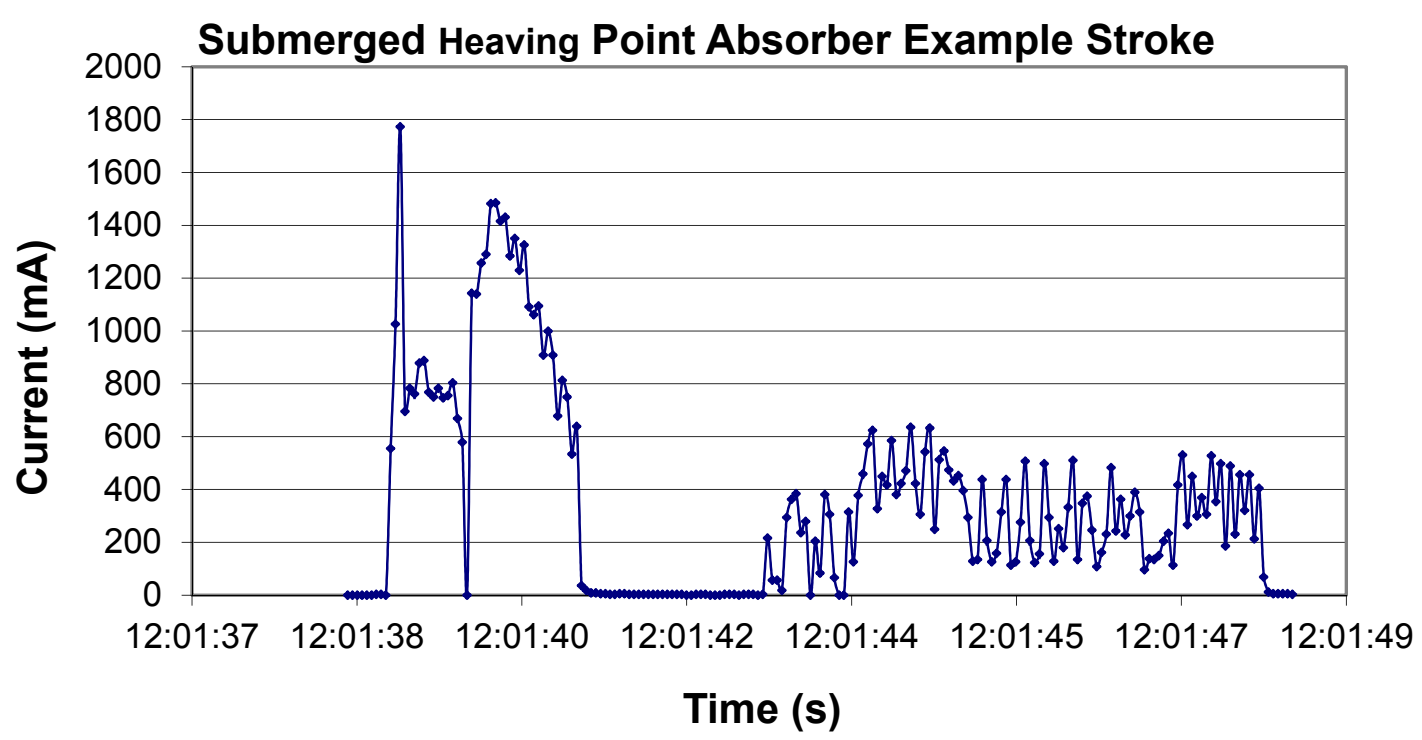

Figure 8. Example stroke with a 2 second extension time and a 5 second retraction time 
The device operated as intended and can achieve further increases in power production with the selection of a more suitable rotary generator.

\section{CONCLUSION}

The assessment of all energy sources within the oceanic environment through a literature review resulted in the determination that wave energy is the most suitable for harvesting. This was due to a high energy density, minimal periods of energy stagnation, and wave prevalence within oceanic environments off the coast of Australia.

The generation of concept design modelling and the subsequent simulation and evaluation against design criteria resulted in the final designs of two wave energy harvesting devices. These were the surface buoy energy converter and the heaving point absorber.

Both devices were manufactured and tested within a laboratory setting. After completing the laboratory testing, the surface buoy energy converter was found to have operated as intended but produced lower than expected power generation. This was due to a number of linear generator deficiencies within the design. Further design iterations implementing observations concluded during the testing would result in greater power generation potential. The submerged heaving point absorber operated as intended. The device has the potential to power a range of sensory equipment with the selection of an appropriate generator.

\section{ACKNOWLEDGEMENTS}

The Authors would like to thank John van Velzen from the industry sponsor, the Defence Science and Technology Organisation (DSTO), for ongoing assistance in the development of design concepts, financial support and allowing access to manufacturing and testing equipment.

The University of Adelaide's Electrical Workshop, in particular Phillip Schmidt, provided valuable technical assistance, for which we are very grateful. Phillip Schmidt has allowed the use of surplus materials, testing and manufacturing equipment for the production of prototype components.

\section{REFERENCES}

Aubrecht, J A 2005, Energy: Physical, Environmental, and Social Impact, Addison Wesley

Behrens, S, Heyward, J, Hemer, M, Osman, P 2011, 'Assessing the wave energy converter potential for Australian coastal regions', Renewable Energy, vol. 43, pp. 210-217

Clement, P, McCullen, A, Fiorentino, F, Gardner, K, Hammarlund, G, Lemonis, G, Lewis, T, Nielsen, K, Petroncini, S, Pontes, M, Schild, P, Sjostrom, B, Sorensen, C, Thorpe, T 2002, 'Wave Energy in Europe: current status and perspectives', Renewable and Sustainable Energies, vol. 6, pp. 405-431

Falnes, J 2007, ‘A review of wave-energy extraction', ScienceDirect, vol. 20, pp. 185-201

Folley, M, Whittaker, T 2009, 'Analysis of the nearshore wave energy resource', Renewable Energy, vol. 34, pp. $1709-1715$

Herbich, J 2000, Handbook of coastal engineering, Mcgraw-Hill professional

Phillips, M 1977 The dynamics of the upper Ocean, Cambridge University Press, ISBN 0-521-29801-6

Seabased 2012, About Wave Energy, Seabased, viewed $19^{\text {th }}$ October 2012, http://www.seabased.com/index.php?option $=$ com content $\&$ view $=$ article\&id $=73 \&$ Itemid $=72$

Torsethaugen, K 1982, 'The Norwegian “wave climate mapping”' programme', Proceedings of the second international symposium on wave energy utilization. Norway: Tapir, Trondheim, pp. 81-97

Vining, J 2005, 'Ocean Wave Energy Conversion', unpublished study report, University of Wisconsin Madison.

Yigone China 2012, Ball Screw, Nanjing Technical Equipment Manufacture Co., Ltd, viewed $2^{\text {nd }}$ October 2012, http://www.linearguidechina.com/2-10-ball-screw.html 